\title{
Rhabdomyolysis plus Hypocalcemia and Diabetic Ketoacidosis as Concurrent Rare COVID-19 Manifestations
}

\author{
Maryam Heidarpour $\mathbb{D}^{1},{ }^{1}$ Mehrbod Vakhshoori $\mathbb{D}^{2},{ }^{2}$ Mohammad Ali Haghighatpanah $\mathbb{D}^{3},{ }^{3}$ \\ Leila Ashrafi $\mathbb{D}^{1},{ }^{1}$ Farzin Khorvash $\mathbb{D}^{4},{ }^{4}$ and Bijan Iraj $\mathbb{D}^{1}$ \\ ${ }^{1}$ Isfahan Endocrine and Metabolism Research Center, Isfahan University of Medical Sciences, Isfahan, Iran \\ ${ }^{2}$ Heart Failure Research Center, Cardiovascular Research Institute, Isfahan University of Medical Sciences, Isfahan, Iran \\ ${ }^{3}$ Isfahan University of Medical Sciences, Isfahan, Iran \\ ${ }^{4}$ Acquired Immunodeficiency Research Center, Isfahan University of Medical Sciences, Isfahan, Iran
}

Correspondence should be addressed to Bijan Iraj; bijaniraj@gmail.com

Received 28 December 2020; Revised 23 February 2021; Accepted 27 February 2021; Published 10 March 2021

Academic Editor: Mamede de Carvalho

Copyright (C) 2021 Maryam Heidarpour et al. This is an open access article distributed under the Creative Commons Attribution License, which permits unrestricted use, distribution, and reproduction in any medium, provided the original work is properly cited.

\begin{abstract}
Background. Common manifestations of coronavirus disease 2019 (COVID-19) from its initial official introduction are mostly related to the respiratory system. However, other rarer presentations are reported nowadays. Case Presentations. We reported three cases of COVID-19-infected patients with rhabdomyolysis as well as two other rarer simultaneous signs, including hypocalcemia (Case 1) and diabetic ketoacidosis (DKA) (Case 2). Conclusion. Despite the fact that rhabdomyolysis is an infrequent manifestation of COVID-19, high clinical suspicion is required for proper diagnosis and management of this disease as well as other concurrent rarer presentations, including hypocalcemia and DKA for the prevention of further complications.
\end{abstract}

\section{Introduction}

Coronavirus disease 2019 (COVID-19) emerges as a novel infectious agent with several body organs' involvement. This disease's most common presentations are fever, dry cough, fatigue, or myalgia [1]. However, some patients develop severe symptoms, including shortness of breath, requiring further therapeutic support. On the other hand, rarer manifestations of COVID-19 have been declared.

Rhabdomyolysis is defined as muscle cell destruction resulting in subsequent leakage of cellular components to the bloodstream. This disease is mostly manifested with weakness, myalgia, electrolyte imbalance, myoglobinuria, or even acute kidney injury [2]. However, the exact etiology of rhabdomyolysis among COVID-19 patients needs to be investigated in future studies.

Moreover, hypocalcemia is a common phenomenon among critically ill patients, and it has been reported that calcium plays a pivotal role in the replication mechanisms of some viruses, including severe acute respiratory syndrome (SARS), Middle East respiratory syndrome (MERS), and Ebolavirus [3,4]. For instance, a study done on patients suffering from SARS reported that hypocalcemia was prevalent in $70 \%$ of individuals during the hospitalization duration [4]. Another study investigated the prevalence of hypocalcemia among severely ill COVID-19 patients and figured out that almost two-thirds of them had lower calcium levels and had subsequent worse outcomes [5].

Diabetic ketoacidosis (DKA) is another rare manifestation during the COVID-19 pandemic. Goldman et al. reported that this disorder is prevalent among $1.8 \%$ of admitted patients [6]. Chan et al. performed a case series on six patients with COVID-19 admitted with DKA and/or hyperosmolar hyperglycemic state (HHS) and found that these two glucose imbalance disorders were the primary manifestations in most cases [7]. However, the data are limited, and several future studies in terms of predisposing factors are required. 
Herein, we reported three cases of COVID-19 patients with rhabdomyolysis as well as other concurrent rarer symptoms, including hypocalcemia and DKA.

\section{Case Presentation}

2.1. Case 1. A 22-year-old man with no previous past medical history was referred to the emergency department with severe hypocalcemia (Ca: $5 \mathrm{mg} / \mathrm{dl}$ ) as well as spastic muscles in both upper and lower extremities. Also, he was opium-addicted and used $15 \mathrm{cc}$ of daily methadone. He had severe bloodless diarrhea from 20 days before admission managed with serum therapy. His vital signs were in normal ranges. He was conscious, but he appeared to be pale. He did not have any positive history in terms of nausea, vomiting, cough, or dyspnea. However, he suffered from generalized muscle spasms and pain. There was not any positive finding during heart and lung examinations. His abdomen was nontender, but pitting edema $(2+)$ was observed in the lower extremities. Chvostek's and Trousseau's signs were positive, and his electrocardiogram (ECG) revealed a prolonged QT interval $(\mathrm{QT}=550 \mathrm{msec})$. Due to the high prevalence of COVID-19, lung high-resolution computed tomography (HRCT) was performed and the results were highly suggestive of COVID-19 infection (Figure 1).

In the first hour of admission, intravenous infusion of $10 \%$ calcium gluconate was initiated promptly, and muscle spasms disappeared within four hours. Intravenous calcium administration was then continued based on plasma-calcium and ECG monitoring. The patient had a myoclonic-like seizure with an upward gaze on the first night of hospitalization, while the serum level of calcium was $8.9 \mathrm{mg} / \mathrm{dl}$. The result of the requested brain CT was negative. Unfortunately, the seizure recurred, and intravenous diazepam was prescribed. On the third day of admission, the calcium concentration reached $9.1 \mathrm{mg} / \mathrm{dl}$, and the QT interval was corrected $(\mathrm{QT}=405 \mathrm{msec})$. Other laboratory data collected during the admission time were as follows: lactic acid dehydrogenase $(\mathrm{LDH})$ of $1740 \mathrm{U} / \mathrm{L}$, creatine phosphokinase $(\mathrm{CPK})$ of $1840 \mathrm{U} / \mathrm{L}$, potassium of $6.9 \mathrm{mEq} / \mathrm{l}$, sodium of $142 \mathrm{mEq} / \mathrm{l}$, blood sugar of $234 \mathrm{mg} / \mathrm{dl}$, albumin of $3.2 \mathrm{~g} / \mathrm{dl}$, aspartate aminotransferase (AST) of $261 \mathrm{IU} / \mathrm{L}$, alanine aminotransferase (ALT) of $110 \mathrm{IU} / \mathrm{L}$, alkaline phosphatase (ALP) of $379 \mathrm{IU} / \mathrm{L}$, parathyroid hormone (PTH) of $145 \mathrm{pg} /$ $\mathrm{ml}, 25$-hydroxy vitamin $\mathrm{D}$ of $32 \mathrm{ng} / \mathrm{mL}$, blood urea nitrogen (BUN) of $19 \mathrm{mg} / \mathrm{dl}$, and creatinine $(\mathrm{Cr})$ of $1.2 \mathrm{mg} / \mathrm{dl}$.

His laboratory findings favored rhabdomyolysis, and fluid therapy was started promptly with concurrent seizure as well as hypocalcemia management to avoid acute kidney injury. Unfortunately, the patient had a cardiac and respiratory arrest, and cardiopulmonary resuscitation (CPR) was implemented, and he was connected to a ventilator. However, on the fourth day of hospitalization, the patient could not be resuscitated after the second cardiac arrest attack.

2.2. Case 2. A 36-year-old man was referred to the emergency department with two days of polyuria, polydipsia, nausea, and vomiting. His medical history was positive for psychosis, and he consumed olanzapine, lorazepam, propranolol, and sertraline for the last ten years. He was opiumaddicted and used $20 \mathrm{cc}$ of daily methadone. At admission, he was confused and lethargic with normal vital signs except for high temperature $\left(T=38.5^{\circ} \mathrm{C}\right)$. Besides dehydration, other physical examinations did not reveal any positive findings. His blood sample was taken and showed a blood sugar of $500 \mathrm{mg} / \mathrm{dl}, \mathrm{pH}$ of 7 , and $\mathrm{HCO}_{3}$ of $2.5 \mathrm{mEq} / \mathrm{l}$. With the assumed diagnosis of DKA, insulin, tazocin, and serum therapy was initiated. The patient's general condition and blood sugar levels were improved during the next day, and the $\mathrm{HCO}_{3}$ concentration was raised to $9.5 \mathrm{mEq} / \mathrm{l}$. On the third day of admission, his $\mathrm{HCO}_{3}$ was $11 \mathrm{mEq} / \mathrm{l}$. However, insulin infusion was held due to a lower potassium level. The patient suffered from dyspnea and shortness of breath on the next day and was connected to the ventilator. HRCT was requested, and the results were highly suggestive of COVID19 infection (Figure 2). Additional laboratory data were as follows: potassium of $7 \mathrm{mEq} / \mathrm{l}$, sodium of $138 \mathrm{mEq} / \mathrm{l}, \mathrm{LDH}$ of $1296 \mathrm{U} / \mathrm{L}, \mathrm{CPK}$ of $5130 \mathrm{U} / \mathrm{L}$, phosphorus of $2 \mathrm{mg} / \mathrm{dl}$, and $\mathrm{Cr}$ of $5.3 \mathrm{mg} / \mathrm{dl}$.

Prompt therapeutic measurements were done, and because of decreased urine output, a temporary dialysis catheter was inserted, and he underwent hemodialysis. During the hospital admission, he experienced an attack of hypotension managed with norepinephrine as well as hydrocortisone. An abdominal computed tomography (CT) scan was performed due to increased amylase (142 U/L) and lipase (351 U/L) levels. The findings of imaging studies and the analysis of ascites fluid favored pancreatitis (Figure 3(a)). Moreover, this CT was highly suggestive of concurrent splenic vein thrombosis (Figure 3(b)). All appropriate therapies were implemented, and most of the laboratory data were improved consequently. The hemodialysis course was discontinued, and he was discharged from the hospital after the second abdominal CT examination indicated resolved signs of pancreatitis. On the 45th day, his general condition was good. He received insulin twice a day and daily orally administered warfarin with a dosage of $5 \mathrm{mg}$ adjusted to keep the patient's international normalized ratio (INR) value of over 2 .

2.3. Case 3. A 47-year-old woman came to the hospital with respiratory symptoms, including fever, cough, and dyspnea, for two weeks. She had a long history of diabetes mellitus, hypertension, hypothyroidism, and asthma, which had been medically controlled. Other vital signs were in normal ranges. She did not complain of muscle pain. Due to high suspicion for COVID-19 infection, she was tested, and the results of reverse transcriptase-polymerase chain reaction (RT-PCR) and HRCT were suggestive of this viral infection (Figure 4). She was transferred to the infectious ward. Her laboratory findings showed $\mathrm{LDH}$ of $5580 \mathrm{U} / \mathrm{L}, \mathrm{CPK}$ of $16480 \mathrm{U} / \mathrm{L}, \mathrm{Cr}$ of $1.7 \mathrm{mg} / \mathrm{dl}$, AST of $951 \mathrm{IU} / \mathrm{L}$, ALT of $874 \mathrm{IU} /$ $\mathrm{L}$, and ALP of $394 \mathrm{IU} / \mathrm{L}$. With the assumed diagnosis of rhabdomyolysis, serum therapy was prescribed. The shortness of breath worsened on the fifth day and resulted in the New York Heart Association (NYHA) class of IV worsening 


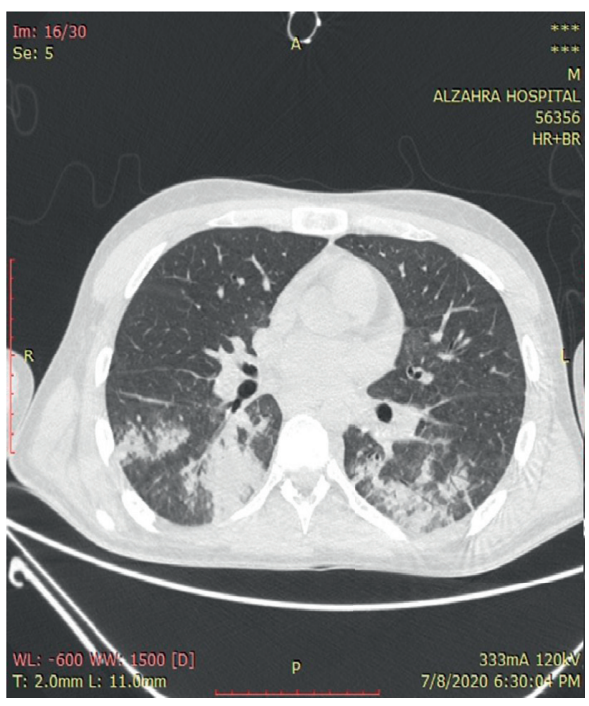

(a)

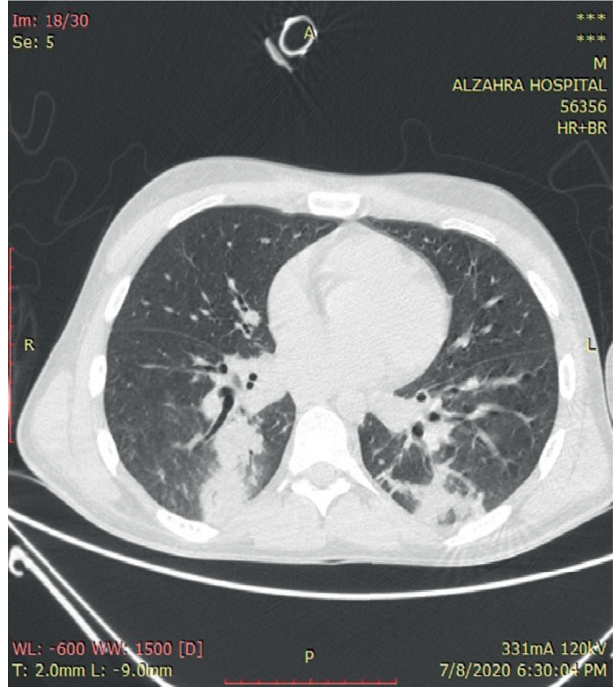

(b)

FIGURE 1: Multifocal peribronchial consolidation and diffuse ground-glass opacities.

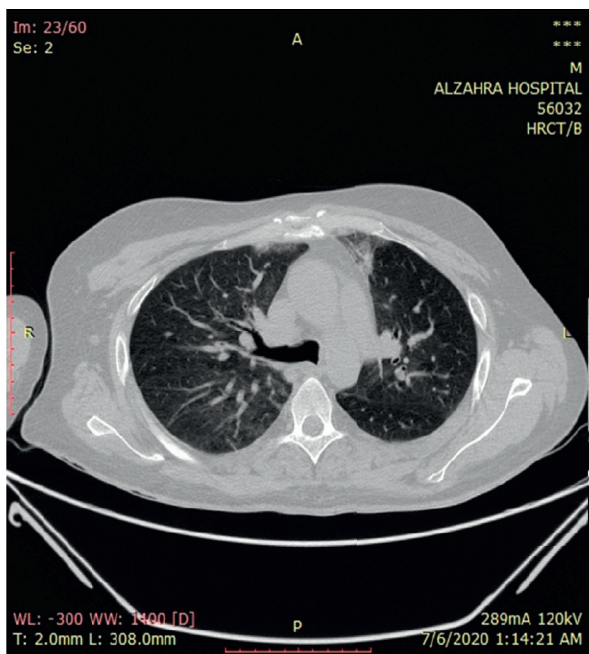

(a)

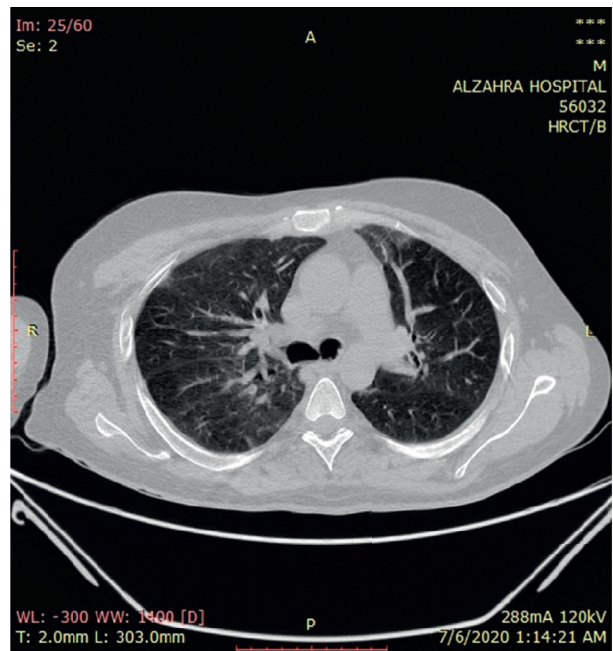

(b)

FIGURE 2: Bilateral and peripheral ground-glass pulmonary opacities.

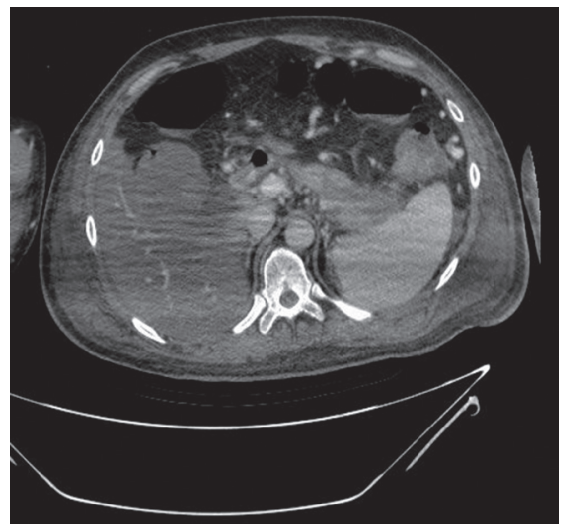

(a)

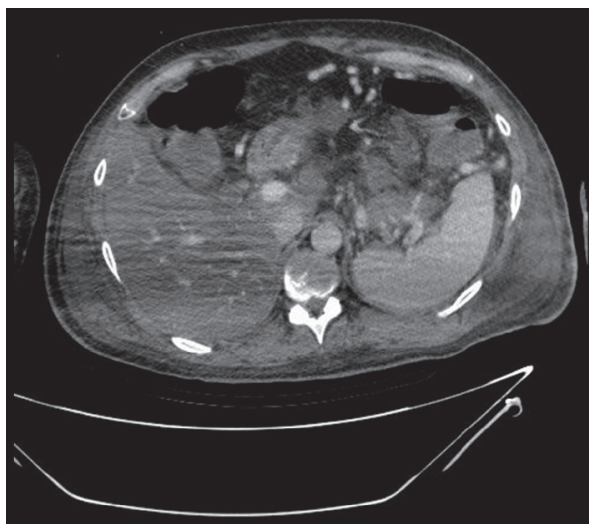

(b)

FIGURE 3: Heterogeneity and edema of the pancreatic tail with low enhancement (a) and splenic vein thrombosis with multiple collaterals (b). 


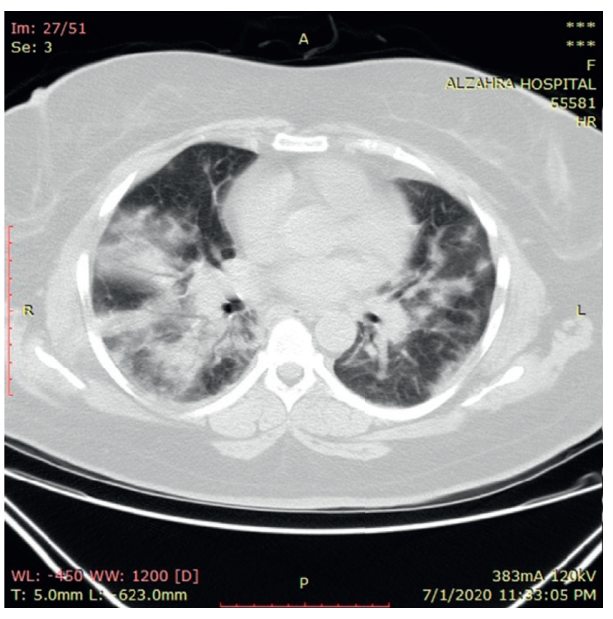

(a)

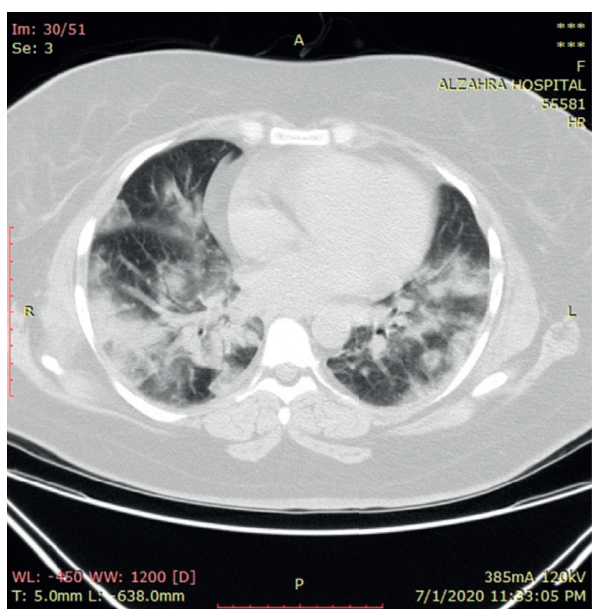

(b)

FIgURE 4: Multifocal multilobular consolidation and ground-glass opacities.

cardiac function with severe hemoglobin oxygen desaturation due to bronchial secretions. Despite receiving 100\% oxygen via a face mask, oxygen saturation only reached $80 \%$. Therefore, the decision was made in order to intubate the patient. She was transferred to the intensive care unit (ICU), and the proper treatments were continued. Her laboratory findings were improved during the next week (LDH: $1204 \mathrm{U} /$ L, CPK: 560 U/L, Cr: 0.7 mg/dl, AST: 135 IU/L, ALT: 234 IU/ L, and ALP: $562 \mathrm{IU} / \mathrm{L})$. The patient remained stable hemodynamically; however, she became unable to wean from a ventilator secondary to probable acute respiratory distress syndrome (ARDS). Unfortunately, in the third week, she had a sudden cardiac arrest. She underwent cardiorespiratory resuscitation but could not be resuscitated from her pulseless electrical activity arrest and died.

\section{Discussion}

Since the emergence of COVID-19, several other manifestations rather than pulmonary symptoms have been reported. Here, we presented three cases of confirmed COVID-19 infection with concomitant rhabdomyolysis as well as hypocalcemia and DKA. Rhabdomyolysis is a life-threatening syndrome characterized by skeletal muscle cell breakdown and leakage of cellular components, including potassium, phosphorus, CPK, and myoglobin, to the bloodstream. The typical triad of this disease consisted of myalgia, weakness, and dark-colored urine. However, less than $10 \%$ of patients experienced the classic triad [8]. Multiple etiologies have been suggested, including drug side effects, trauma, autoimmune status, exertion, ischemia, and infection [9]. Common viral causes include influenza $\mathrm{A}$ and $\mathrm{B}$, herpes simplex virus, Epstein-Barr virus, cytomegalovirus, human immunodeficiency virus, and enteroviruses [10]. Even though our patients were not tested for other viral diseases, clinical status and laboratory data confirmed COVID-19 infection. While the exact pathophysiological mechanism attributed to rhabdomyolysis occurrence in COVID-19 infection has yet to be investigated, several theories have been declared. Due to the genomic similarity between SARS and COVID-19 and the ability of SARS to directly infect muscle cells, it probably seems that this new infective agent can infect myocytes. The formation of immune complexes and their deposition because of cross-reactivity between muscle cells and viral antigens might be responsible for muscular damage. Massive cytokine production and cytotoxic T-cell activation could be categorized as other possible causes in this regard $[10,11]$. On the other hand, other rare comanifestations of COVID-19-induced rhabdomyolysis must be considered. Case 1 was admitted with severe hypocalcemia as the initial presentation. Although the patient had myalgia and experienced seizure, which might be due to rhabdomyolysis complications, the direct virus effect for causing these presentations should be considered. Liu et al. performed a retrospective study to assess the predictive effect of hypocalcemia among patients with severe COVID-19. They figured out that $62.6 \%$ of patients had low calcium levels, which was associated with poorer outcomes [5]. Hypocalcemia is quite common in viral infections and among critically ill patients. The possible factors include malnutrition, decreased intestinal absorption of calcium, cell membrane damage due to hypoxia and calcium influx, vitamin D deficiency, or medication interaction. Moreover, serum calcium is mainly bound to albumin, and hypocalcemia could occur in favor of reduced albumin levels. However, cytokine production during COVID-19 infection is reported to inhibit PTH secretion, and impaired response to PTH might be responsible for the induction of hypocalcemia [12]. Several studies are required to evaluate the exact pathophysiological mechanism responsible for hypocalcemia among COVID-19-infected individuals. Overall, in our case, it seems that impaired response to PTH, malnutrition, and calcium influx were possible causes of hypocalcemia.

In terms of DKA that occurred in Case 2, several points should be considered. The infection itself might be responsible for the induction of DKA [7]. Also, abnormal blood glucose has been reported to be associated with worse outcomes in the COVID-19 pandemic. Although the data are still limited, further investigations for the diagnosis of the 
exact mechanism are required. The Coronaviridae family has the tropism for both exocrine and endocrine pancreatic cells. Like SARS, angiotensin-converting enzyme 2 (ACE2) has been suggested to be a receptor for COVID-19 S-protein [13]. This receptor has been expressed in pancreatic betacells in addition to pulmonary cells [14]. The primary role of ACE2 is the degradation of angiotensin 2 to angiotensin1-7. Therefore, downregulation of the ACE2 receptor during COVID-19 leads to increased angiotensin 2 concentration resulting in reduced blood supply to pancreatic cells and subsequently delayed insulin secretion, which ultimately results in hyperglycemia and even DKA occurrence $[14,15]$. However, whether the sudden onset alterations of glucose metabolism in severe COVID-19 will be persisted or remitted is still unclear. The presence of this receptor on the exocrine pancreas might explain the incidence of pancreatitis in Case 2. It seems that pancreatic involvement during COVID-19 should be further addressed in clinical settings.

In conclusion, our findings from this case report indicate that rhabdomyolysis could be categorized as one of the complications of COVID-19, and further suspicion for proper diagnosis and therapeutic interventions should be performed. Furthermore, other simultaneous rare manifestations, including hypocalcemia or DKA, must be comprehensively addressed in future studies.

\section{Abbreviations}

COVID- Coronavirus disease 2019

19:

SARS: $\quad$ Severe acute respiratory syndrome

MERS: $\quad$ Middle East respiratory syndrome

DKA: Diabetic ketoacidosis

HHS: Hyperosmolar hyperglycemic state

ECG: $\quad$ Electrocardiogram

HRCT: High-resolution computed tomography

LDH: $\quad$ Lactic acid dehydrogenase

CPK: $\quad$ Creatine phosphokinase

AST: Aspartate aminotransferase

ALT: Alanine aminotransferase

ALP: $\quad$ Alkaline phosphatase

PTH: Parathyroid hormone

BUN: Blood urea nitrogen

Cr: Creatinine

CPR: Cardiopulmonary resuscitation

CT: $\quad$ Computed tomography

INR: International normalized ratio

RT-PCR: Reverse transcriptase-polymerase chain reaction

NYHA: New York Heart Association

ICU: Intensive care unit

ARDS: Acute respiratory distress syndrome

ACE2: $\quad$ Angiotensin-converting enzyme 2.

\section{Data Availability}

The datasets generated and/or analyzed during the current study are not publicly available due to confidential issues but are available from the corresponding author on reasonable request.

\section{Consent}

Written informed consent was obtained from the patient or patient's next of kin (when the death happened) for publication of this case report and any accompanying images.

\section{Disclosure}

Maryam Heidarpour and Mehrbod Vakhshoori are considered co-first authors.

\section{Conflicts of Interest}

None of the authors had any personal or financial conflicts of interest.

\section{Authors' Contributions}

M. H., B. I., and F. K. contributed to study concept and design and provided administrative, technical, and material support. M. H., MA. H., and L. A. were responsible for the acquisition of data. M. V., MA. H., and L. A. drafted the manuscript. M. V., B. I., M. H., and F. K. critically revised the manuscript for valuable intellectual content. B. I., M. H., and F. K supervised the study. M. H. and M. V. contributed equally to this manuscript.

\section{References}

[1] L. Di Filippo, A. M. Formenti, P. Rovere-Querini et al., "Hypocalcemia is highly prevalent and predicts hospitalization in patients with COVID-19," Endocrine, vol. 68, no. 3, pp. 1-4, 2020.

[2] Z. Al-Ismaili, M. Piccioni, and M. Zappitelli, "Rhabdomyolysis: pathogenesis of renal injury and management," Pediatric Nephrology, vol. 26, no. 10, pp. 1781-1788, 2011.

[3] J. K. Millet and G. R. Whittaker, "Physiological and molecular triggers for SARS-CoV membrane fusion and entry into host cells," Virology, vol. 517, pp. 3-8, 2018.

[4] L. Nathan, A. L. Lai, J. K. Millet et al., "Calcium ions directly interact with the ebola virus fusion peptide to promote structure-function changes that enhance infection," ACS Infectious Diseases, vol. 6, no. 2, pp. 250-260, 2019.

[5] J. Liu, P. Han, J. Wu, J. Gong, and D. Tian, "Prevalence and predictive value of hypocalcemia in severe COVID-19 patients," Journal of Infection and Public Health, vol. 13, no. 9, pp. 1224-1228, 2020.

[6] N. Goldman, D. Fink, J. Cai, Y.-N. Lee, and Z. Davies, "High prevalence of COVID-19-associated diabetic ketoacidosis in UK secondary care," Diabetes Research and Clinical Practice, vol. 166, Article ID 108291, 2020.

[7] K. H. Chan, D. Thimmareddygari, A. Ramahi, L. Atallah, N. G. Baranetsky, and J. Slim, "Clinical characteristics and outcome in patients with combined diabetic ketoacidosis and hyperosmolar hyperglycemic state associated with COVID19: a retrospective, hospital-based observational case series," Diabetes Research and Clinical Practice, vol. 166, Article ID 108279, 2020. 
[8] R. Zutt, A. J. Van Der Kooi, G. E. Linthorst, R. J. A. Wanders, and M. De Visser, "Rhabdomyolysis: review of the literature," Neuromuscular Disorders, vol. 24, no. 8, pp. 651-659, 2014.

[9] W. H. Bagley, H. Yang, and K. H. Shah, "Rhabdomyolysis," Internal and Emergency Medicine, vol. 2, no. 3, pp. 210-218, 2007.

[10] N. F. Crum-Cianflone, "Bacterial, fungal, parasitic, and viral myositis," Clinical Microbiology Reviews, vol. 21, no. 3, pp. 473-494, 2008.

[11] Y. Liang, M.-L. Wang, C.-S. Chien et al., "Highlight of immune pathogenic response and hematopathologic effect in SARS-CoV, MERS-CoV, and SARS-cov-2 infection," Frontiers in Immunology, vol. 11, p. 1022, 2020.

[12] J. Fong and A. Khan, "Hypocalcemia: updates in diagnosis and management for primary care," Canadian family physician Medecin de famille canadien, vol. 58, no. 2, pp. 158-162, 2012.

[13] M. Hoffmann, H. Kleine-Weber, S. Schroeder et al., "SARSCoV-2 cell entry depends on ACE2 and TMPRSS2 and is blocked by a clinically proven protease inhibitor," Cell, vol. 181, no. 2, 2020.

[14] J.-K. Yang, S.-S. Lin, X.-J. Ji, and L.-M. Guo, "Binding of SARS coronavirus to its receptor damages islets and causes acute diabetes," Acta Diabetologica, vol. 47, no. 3, pp. 193-199, 2010.

[15] P.-O. Carlsson, C. Berne, and L. Jansson, "Angiotensin II and the endocrine pancreas: effects on islet blood flow and insulin secretion in rats," Diabetologia, vol. 41, no. 2, pp. 127-133, 1998. 